\title{
Ethernet Based Remote Monitoring And Control Of Temperature By Using Rabbit Processor
}

\author{
U. SUNEETHA \\ Department of Electronics \\ Sri Krishnadevaraya University \\ Anantapur, INDIA
}

\author{
K.TANVEER ALAM \\ Department of Electronics \\ Sri Krishnadevaraya University \\ Anantapur, INDIA
}

\author{
N.ANJU LATHA \\ Department of Instrumentation Sri \\ Krishnadevaraya University \\ Anantapur, INDIA
}

\author{
B.V.S.GOUD \\ Department of electronics and Instrumentation \\ Acharya Nagarguna University \\ Guntur, INDIA
}

\author{
B.RAMAMURTHY \\ Department of Instrumentation \\ Sri Krishnadevaraya University Anantapur, INDIA
}

\begin{abstract}
Networking is a major component of the processes and control instrumentation systems as the network's architecture solves many of the Industrial automation problems. There is a great deal of benefits in the process of industrial parameters to adopt the Ethernet control system. Hence an attempt has been made to develop an Ethernet based remote monitoring and control of temperature. In the present work the experimental result shows that remote monitoring and control system (RMACS) over the Ethernet.
\end{abstract}

Keywords- RMACS; Control system; Ethernet.

\section{INTRODUCTION}

The exponential growth of Internet and computer technology enables the development of complex, hybrid systems which offers greater concern in maintenance and has more flexibility in servicing and fault finding $[1,2]$. With the advanced technology industries are interested in automation by introducing remote monitoring and control system for the measurement control of industrial process parameters very precisely and accurately for the quality products. The Ethernet provides an inexpensive gateway through which to data transfer for real-time interaction of the remote monitoring and control of the parameter give many advantages.

RMACS is an effective on-line monitoring and control system and to transmit the real time data on to the terminal [3]. The RMACS is an effective also to analyze, manage and feedback the remote information and it combines the most advanced science and technology, in the field of communication technology, Internet technology and other areas.

The main objective of the present work is to develop a system for remote monitoring and control of temperature over the Ethernet. Accurate measurement and control of temperature is essential nearly in all chemical processes which require some times below than $+1^{\circ} \mathrm{C}$ or $-1^{\circ} \mathrm{C}$ accuracy.
Temperature is measured using different methods currently in use: thermocouples (TC) [4], thermister [5, 6], resistance temperature detectors (RTD) [7] and integrated circuits (IC). Most of the temperature sensors produce an analog at present which is further convert to digital form using A/D converter interfacing with the processing like microprocessor, microcontroller and PC or their combination.

\section{HARDWARE IMPLEMENTATION}

The block diagram and schematic diagram of the hardware system RMACS [8] is shown in the fig.1 and fig.2 respectively. The system consists of the following units. They are Temperature sensor (LM 35), signal conditioning unit (MAX 186), Rabbit processor, Ethernet, RTL8019AS, Relay control device (FAN) and PC.

In the system Rabbit 3000 processor is used to measure and control the parameter. Input port is used to sense the temperature and output port is used to control the process. Ethernet provide communication capability to the system. Temperature bath is used to set different temperatures. Sensor is placed within the temperature bath so that its output is being monitored by the system. The signal conditioned by MAX 186 , which will be able to provide the signals to be properly detected by the processor. The data logging is achieved continuously by Rabbit processor to the PC via MAX 232 (Level converter). By implement the software program developed on the PC update the database.

\section{A. Temperature Sensor (LM35)}

The LM35 series are precision integrated-circuit temperature sensor. It is three-terminal device produces an electric voltage proportional to degree Celsius $\left(10 \mathrm{mv} /{ }^{\circ} \mathrm{C}\right)$. These sensors are capable to measure temperature below $0^{\circ} \mathrm{C}$ by using a pull down resistor $\left( \pm 1^{\circ} \mathrm{C}\right.$ from $-55^{\circ} \mathrm{C}$ to $+150^{\circ} \mathrm{C}$ vs. $\pm 3^{\circ} \mathrm{C}$ from $-20^{\circ} \mathrm{C}$ to $+100^{\circ} \mathrm{C}$ ). Thus LM 35 has an advantage over linear temperature sensors calibrated in degree Kelvin, as the user is not required to subtract a large constant voltage 
from its output to obtain convenient centigrade scaling and not require any external calibration.

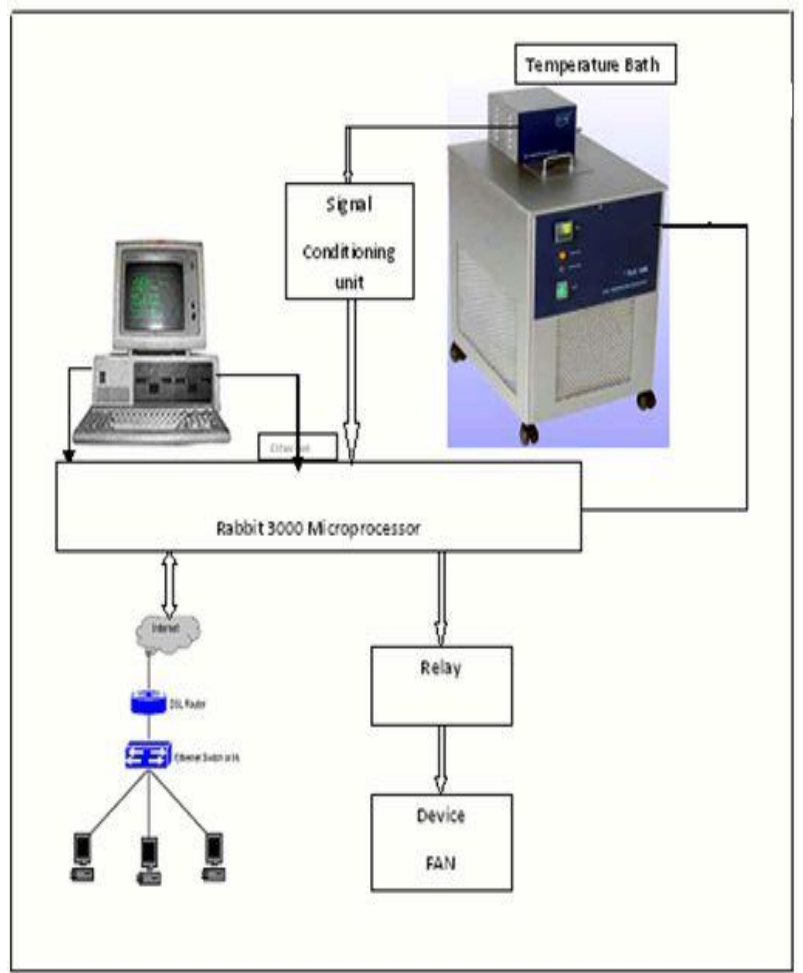

Fig.1 Block diagram of RMACS for Temperature with Ethernet

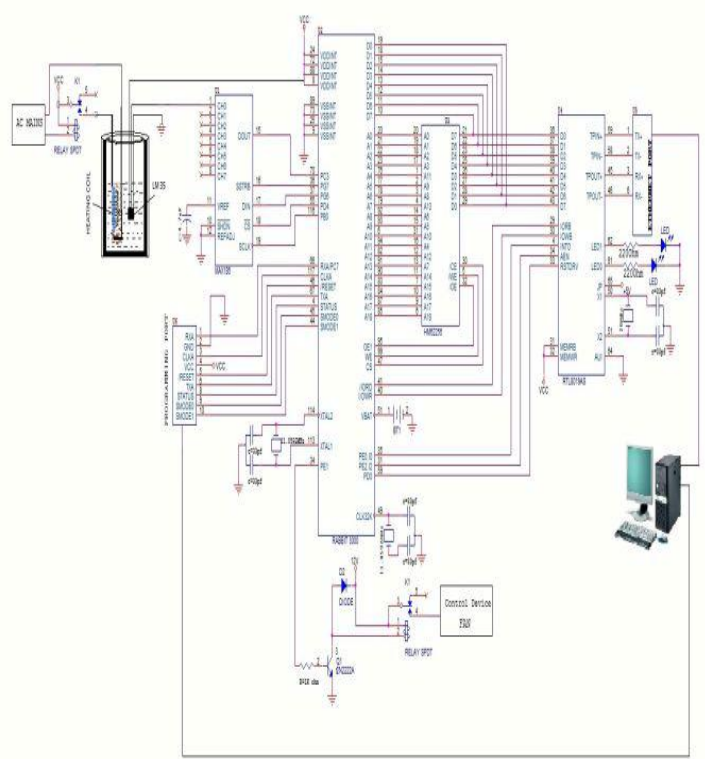

Fig.2 Schematic diagram of RAMACS for temperature with Ethernet.

\section{B. Control device}

In the present system a FAN is used to control the temperature precisely by switch on/off to reduce temperature of the coil by connecting one of the I/O lines of the processor.

\section{Signal conditioning unit (MAX 186)}

The signal conditioning unit consists of an ADC. The sensor output is typically connected to the ADC(MAX 186) which has 12-bit analog to digital converter combines an 8channel multiplexer and serial interface together with high conversion speed and ultra-low power consumption. The analog inputs are software configurable for unipolar/bipolar and single-ended/ differential operation.

\section{Rabbit processor}

In the present study a 16-bit Rabbit processor is used as a processing tool for the remote measurement and control of temperature. It has 8-bit external data bus and an 8-bit internal data bus, address lines (A0-A18) and the data lines (D0-D7), the onboard $512 \mathrm{~K}$ flash memory and 512K SRAM chips, EPROM. 1Mbyte serial flash is also available to store data on Web pages. Rabbit processor is having six serial ports [9] for asynchronous communication.

\section{E. Ethernet}

The word NETWORK [10] implies a linkage between two or more computing devices together for the purpose of sharing data. The network can be categorized as local area networks (LANs) and wide area networks (WAN)[11,12]. Ethernet is a family of frame based computer network technologies for local area networks (LANs) with the data transfer rate as high as $10 \mathrm{Mbps}$. The principle to access the Ethernet is carrier sense Multiple access with collision detection (CSMA/CD) $[13,14]$. The Ethernet is having two layers one is physical layer which converts the data into electrical signals. Second is data link layer it is further divided into two sublayers. One is logic link control (LLC) which is responsible for flow and error control. Next is media access control (MAC) which is responsible for the operation of CSMA/CD access method. Now a days, the most popular protocol is TCP/IP[15]. TCP protocol is said to be connection oriented and provides a reliable service.

\section{F. RTL8019AS}

It is highly integrated Ethernet card (NIC: network interface card) implemented with a plug and play NE 2000 compatible full duplex and power down features having built in 16 byte SRAM in a single chip Consisting PPT (point to point protocol) protocol for logic link layer.

This will carry the signals to data link layer played to the RJ-45 Ethernet jack.

\section{G. Personal computer}

Personal computers have high-speed Internet allowing access to the World Wide Web. In the present work the data logging is achieved continuously from the Rabbit processor to personal computer via MAX 232.

Data is received by implementing the software program developed for the present work which updates the data by using html. On the client side this html file is transferred by using hypertext transfer protocol (HTTP). 


\section{SOFTWARE DEVELOPMENT}

The code is developed for RMACS using the Dynamic C software with a certain socket libraries and http libraries. By initializing this library functions we can develop server software. Library files with Dynamic C provide a full range of serial communication support. The temperature monitored on html document. On client side html file can be browsed with Internet browser which can display the temperature on http service. The html file exists within the http services. If the temperature variation takes place the html file is updated and http server refresh the data. In this way Remote Monitoring and Control of temperature is done by using rabbit processor. The flowcharts depicting the monitoring and the control temperature is shown in fig.3.

\section{RESUlts AND Discussions}

Main object of the present work is to develop an RMACS for the industrial process parameter like temperature on real time basis with Ethernet which is tiny, rugged, low cost and low power consumption ideally suited for industrial control applications. The graphical representation of real-time vs temperature for different set points is shown in figure 4 and photograph of the remote monitoring system with Ethernet Figure 5.

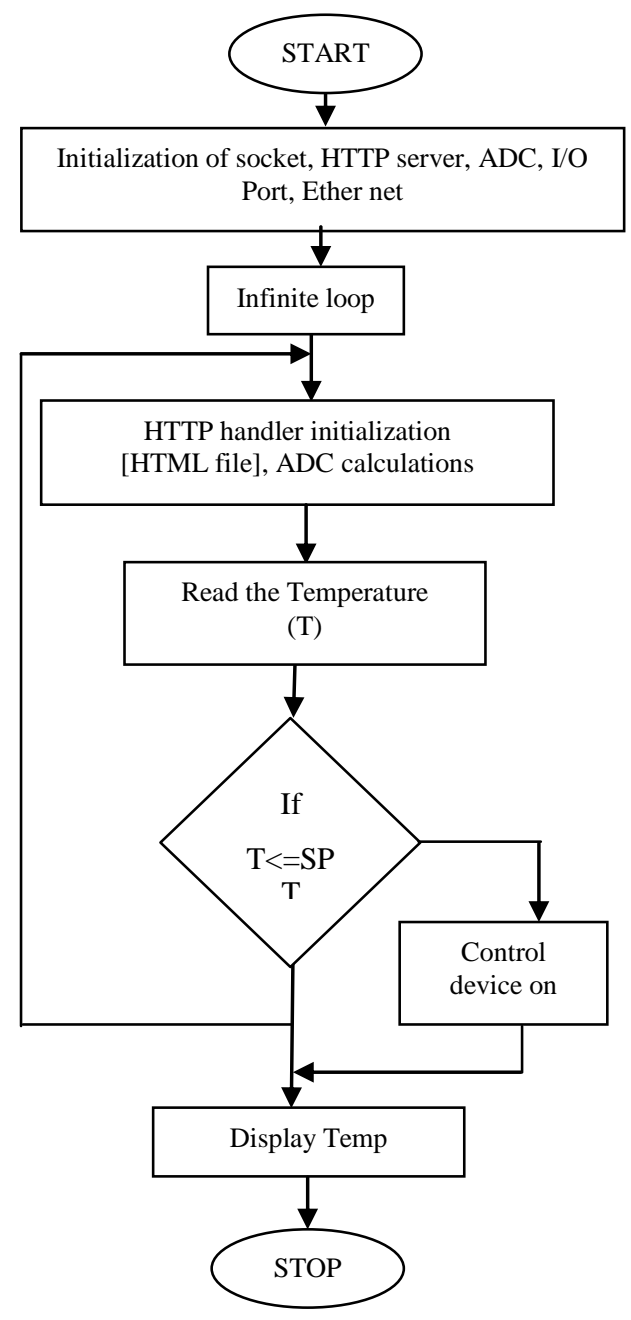

Fig 3: Flowchart for RMACS of temperature with Ethernet.

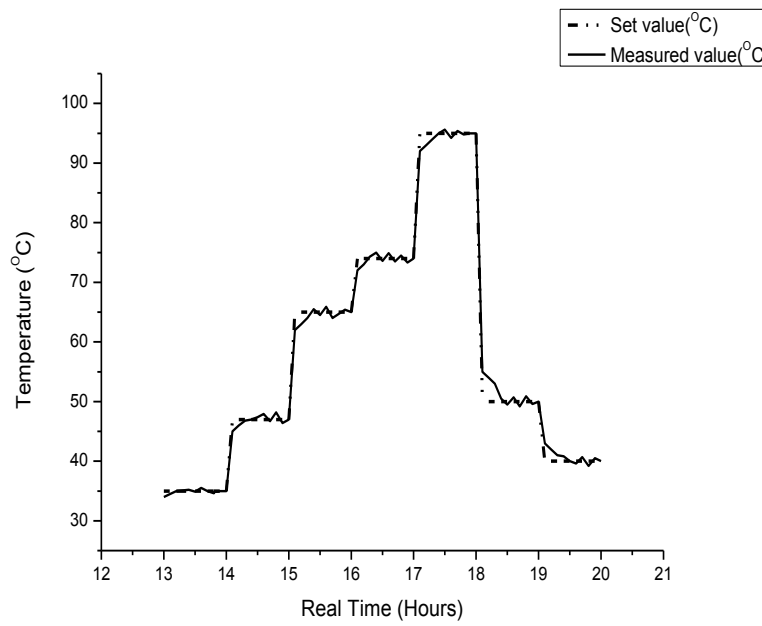

Fig4: Graphical representation of RMACS for temperature with Ethernet.

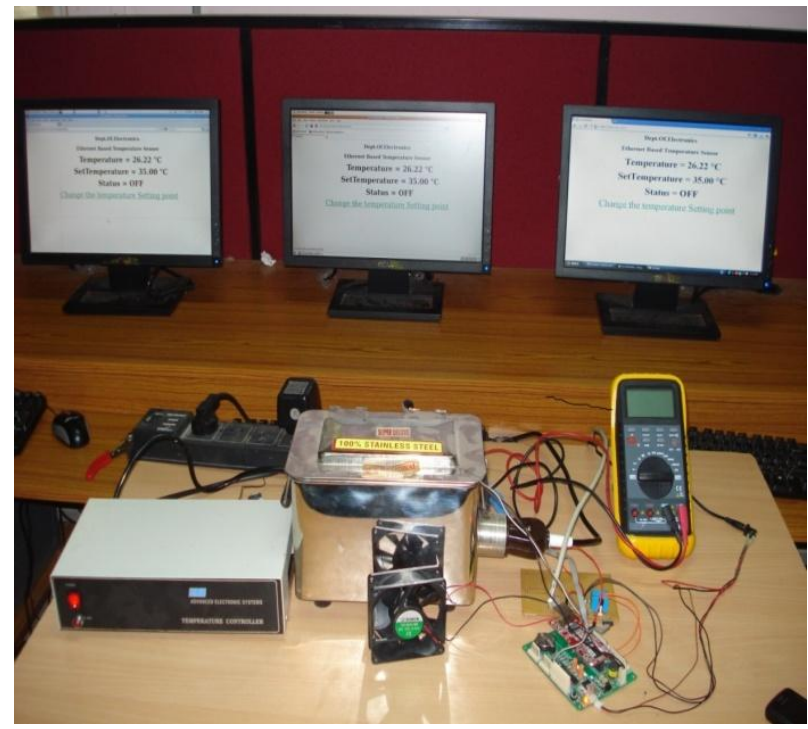

Fig.5. photograph of RMACS for temperature with Ethernet.

\section{CONCLUSION}

A low cost approach of the present developed work is novel and has achieved the target to control process parameter like temperature remotely using the Ethernet. The system is low cost as compared to the previously existing systems like GSM, Wifi with an accuracy of +or $-1{ }^{\circ} \mathrm{C}$.

\section{REFERENCES}

[1] P. line, "Design and implementation of an internet-based virtual lab system for learning support", Proc.5thIEE International conf on Advanced learning Technologies, Kaohsiung, Taiwan, 005, pp.295-296.

[2] Bagnasco \& A.Scapolla, "Agrid of remote laboratory for teaching electronics", $2^{\text {nd }}$ International LeGE-WG workshop on e learning and grid technologies: a fundamental challenge for Europe, Paries, 2003.

[3] N.C.Corbett, "Remote Monitoring and Control of advanced gas turbines" Computing and control Engineering Journal, April 2001

[4] Application Note AN107, "Practical Thermocouple Temperature Measurements", Dataforth Corporation.

[5] RTD Measurement and Theory www.omega.com / temperature /z/thertd .html.

[6] N .Mondal, On Certain Topics in Temperature Measurement Using Thermistors, MEE Thesis, Jadavpur University, 1996. 
[7] X.LIN, G.HUBBARD, Sensor and Electronic Biases/Errors in Air Temperature Measurements in Common Weather Station Networks, Journal of atmospheric and oceanic technology, Volume 21, 2004.

[8] Dr. B. Ramamurthy, S. Bhargavi and Dr. R. Shashi Kumar, "Design and implementation Of GSM based Remote Monitoring and Control System for Industrial Process Parameters", IJCSIS, vol.8, no.5, August 2010.

[9] Technical Note TN227, Interfacing External I/O with Rabbit 3000 Designs

[10] H. Ohsaki, M. Murata, and H. Miyahara,"Modeling end-to-end packet delay Dynamics Of the internet using systems identification", proceedings of the International teletraffic congress 17, Salvador da bahia, Brazil, pp.1027-1038, December 2001.

[11] Wright, Gary R and W Richard Stevens TCP/IP Illustrated, vol.2, the implementation. Reading, Wesley, 1998.

[12] BehrouzA.Forouzan TCP/IP protocol suite, vol.3, Basics of protocols.

[13] S.H.Yang, and J.L.Alty,"Development of a distributed simulator for control experiments through the internet", Future generation computer systems. Vol.18, No.4, pp.57-59, April 1999.

[14] S.H.Yang, X.Chen, and J.L.Alty, "design issues and implementation of internet based process control systems", Control Engineering practice, vol.11, No.6.pp.709 720, June 2003.
[15] Hong-Yanli, "Web-based remote monitoring and control for Process plants", International conference on mechine learning and cybernetics, 18- 21 august 2005.

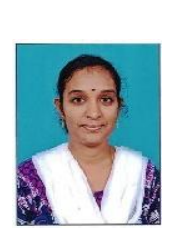

\section{AUTHORS PROFILE}

U.Suneetha is doing P.hd in the Department of Electronics and communications, Sri Krishna Devaraya university, Anantapur. She also working as a Teaching assistant in the Department of Electronics and communications, Sri Krishna Devaraya university, anantapur, Andhrapradesh, India. She having nine years of teaching experience. Her area of interest are Wireless communications and embedded systems.

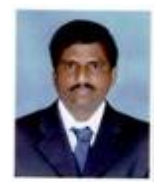

Prof.B.V.S.Goud is currently Head of the department to the department of electronics and instrumentation technology and co-ordinator to the PG Exames, Acharya nagarjuna university, Nagarjuna nagar, guntur. Pior to be he was worked as Deputy manager, Elico plvt ltd. He is the chairman to the board of studies in electronics and instrumentation technology. He is a lifemember of instrumentation socity of india. He has 20 years of teaching experience over 4 years of industrial experience. He has produced several reasearch and review papers in national and international journals. He has complete two major reasearch projects funded by UGC and DAE. He conducted several simphosias, seminors and workshops. 